\title{
Augmentez vos chances d'acceptation de votre manuscrit : Comment répondre aux commentaires des réviseurs
}

\author{
par Mary H H Ensom
}

S

i vous êtes parmi les centaines d'auteurs qui ont soumis un $\bigcirc_{\text {manuscrit au } J C P H}$ au cours des dernières années, vous aurez reçu au moins un avis de décision dans l'une ou plusieurs des catégories suivantes : 1) "rejeté (à l'étape de l'examen préalable) ", 2) «revoir et resoumettre à la révision (à l'étape de l'examen préalable)", 3) "accepté - révision requise" (avec souscatégories "accepté après révision" ou "révisions majeures requises»), 4) «rejeté (après révision par les pairs)» ou 5) «version définitive acceptée ».

À moins que l'article soumis ne l'ait été pour une lettre à la rédaction ou pour une rubrique comme Pleins feux sur la pratique ou Le pour et le contre, pour laquelle vous travailliez déjà avec un rédacteur adjoint, il est peu probable que vous ayez reçu un avis de décision de catégorie $\mathrm{n}^{\circ} 5$. Par conséquent, l'avis comportait probablement des commentaires et des suggestions dont vous deviez tenir compte. L'objectif de cet éditorial est de donner aux auteurs qui ont reçu de tels avis des conseils pour augmenter leurs chances que leur manuscrit soit accepté par le $J C P H$ et, plus particulièrement, sur la façon de répondre aux commentaires des pairs et du rédacteur adjoint attitré. Ces conseils sont basés sur des dizaines d'années d'expérience personnelle en tant qu'auteure, réviseuse, rédactrice adjointe et rédactrice en chef du JCPH, de même que sur mes expériences à titre d'auteure, de réviseuse et de membre de comités de rédaction de nombreuses autres revues biomédicales internationales.

Lorsque vous présentez un manuscrit au JCPH, il est soumis à un examen préalable par le rédacteur en chef ou un rédacteur adjoint avant d'être envoyé à des réviseurs externes. Ainsi, si vous recevez un avis de décision à l'étape de l'examen préalable (c.-à-d. dans la catégorie 1 ou 2), il renfermera typiquement des commentaires du rédacteur en chef ou du rédacteur adjoint seulement.

Un avis de catégorie 1 (rejeté) signifie très probablement que votre manuscrit ne s'inscrit pas dans les domaines privilégiés par le JCPH dont la mission est d'être «une publication internationale respectée tout en étant un instrument majeur pour la dissémination d'information relative à la pratique pharmaceutique axée sur le malade dans les établissements de santé au Canada.» En revanche, si votre manuscrit ne correspondait pas au cadre du JCPH, cela ne signifie pas pour autant qu'il ne trouvera pas sa place dans une autre publication. Dans le doute, n'hésitez pas à discuter préalablement de votre article avec le rédacteur en chef de la publication que vous visez. Comme auteure, j'envoie souvent une demande d'information par courriel au rédacteur en chef d'une revue envisagée afin de confirmer un intérêt pour mon manuscrit. De même, au JCPH nous recevons des courriels exploratoires d'auteurs éventuels et encourageons cette pratique.

Par ailleurs, votre manuscrit peut être rejeté à l'étape de l'examen préalable parce qu'il contient des erreurs de méthodologie que les rédacteurs estiment irrémédiables. Mais il est encore possible que votre article trouve preneur ailleurs, peutêtre même dans la section des lettres à la rédaction du JCPH (où les articles ne sont typiquement pas soumis à la révision par des pairs), s'il est d'intérêt et si vous y incorporez les suggestions des rédacteurs pour l'améliorer.

Un article mal rédigé pourrait tomber dans la catégorie 1 ou 2, selon l'ampleur des faiblesses et les chances de "récupération ". Pour les avis de décision de catégorie $\mathrm{n}^{\circ} 2$ (revoir et resoumettre à la révision), nous demandons habituellement à l'auteur de l'article présentant des faiblesses évidentes de le revoir avant qu'il ne soit soumis aux réviseurs. N'oubliez cependant pas que nous travaillons dans votre intérêt. Parce que nous voulons publier les meilleurs articles qui soient dans notre Journal, nous veillons à vous fournir les commentaires qui vous permettront d'améliorer votre article afin qu'il satisfasse aux normes du Journal avant de passer dans les mains des réviseurs. Il est sage de resoumettre votre article rapidement et de fournir les renseignements supplémentaires ou les révisions qu'on vous demande en regard de chaque commentaire scrupuleusement. 
Si votre article passe l'étape de l'examen préalable, les rédacteurs qui l'ont évalué estiment qu'il a suffisamment de mérite pour être examiné officiellement par des experts du domaine dont l'article est l'objet. Félicitations si votre article atteint cette étape. Bien que les taux d'acceptation pour le JCPH varient selon le type d'article, plus de $50 \%$ des articles envoyés pour examen par les pairs sont éventuellement publiés dans le Journal' ${ }^{1}$. Après que les réviseurs ont remis leurs commentaires, le résultat le plus probable est que l'auteur recevra un avis de décision de catégorie $\mathrm{n}^{\circ} 3$. Règle générale, ce type d'avis comporte des commentaires d'au moins deux réviseurs et du rédacteur adjoint attitré. Nous attendons des auteurs qu'ils donnent suite aux avis de décision dans le même souci de la qualité que nous recherchons chez un bon réviseur; notamment, avec un sens de la promptitude, du tact, de l'organisation, de la critique constructive, de l'objectivité, de la précision, de la spécificité et de l'exhaustivitée

Après avoir reçu un avis de décision de catégorie $\mathrm{n}^{\circ} 3$ accompagné des révisions, vous devriez resoumettre sans tarder une version revue de votre article. Tout retard peut être interprété comme un manque d'enthousiasme de votre part à publier votre article. Si vous avez besoin de plus de temps, vous devriez alors en informer le Journal immédiatement, plutôt que d'attendre après la date butoir à laquelle vous deviez resoumettre votre article. Larticle que vous resoumettez doit être bien organisé et répondre point par point aux commentaires des réviseurs, en prenant soin d'indiquer vos changements dans le texte par page et par numéro de ligne. Vous devez soumettre une version avec des marques de révision (révisions surlignées ou indiquées en mode suivi des modifications) et une version "sans marques " du manuscrit. Vous avez le droit de ne pas être d'accord avec les réviseurs, mais vous devez exprimer vos arguments avec tact; si vous objectez, faites-le de façon respectueuse. Par exemple, vous pouvez faire valoir votre point en commençant ainsi : «J'ai bien pris note du commentaire du réviseur, mais veuillez me permettre d'exposer les raisons pour lesquelles je ne suis pas d'accord. " Vous devriez également toujours répondre de façon constructive aux commentaires des réviseurs. Même si vous estimez qu'ils font fausse route, vous pouvez faire valoir votre point de façon constructive, en l'étayant avec des données probantes. Bien qu'on ne s'attende pas à ce que vous incorporiez chacune des suggestions des réviseurs, vous devriez cependant donner suite scrupuleusement à chaque commentaire dans votre réponse. Bien sûr, vous devrez le faire objectivement, en donnant des références ou en retravaillant certaines sections, comme demandé. En tant qu'auteure, j’avoue que les commentaires des réviseurs m'irritent quelques fois au plus haut point (m'incitant à me demander s'ils ont bien lu mon article!). Cependant, une fois la poussière retombée, je me rends habituellement compte que si un passage était obscur pour un réviseur, il risque de l'être autant pour le futur lecteur. Au bout du compte, mon article s'en est toujours mieux porté après avoir pris en considération les commentaires des réviseurs (que je sois d'accord ou non avec ceux-ci).

Soyez le plus précis et le plus exhaustif possible dans vos échanges avec les réviseurs. Rendez la vie des réviseurs et des rédacteurs plus facile, en mettant à profit toutes les qualités dont il a été question plus haut lorsque vous resoumettez votre article $^{2}$. En effet, si vous retravaillez votre manuscrit en tenant compte exactement des points émis dans l'avis de décision, ce sera difficile pour les rédacteurs de ne pas l'accepter. Il peut arriver que vous receviez des commentaires contradictoires de la part des réviseurs. Si le rédacteur adjoint n’a pas donné de direction quant à l'approche ou à la position à adopter, n’hésitez pas à communiquer avec le Journal (cdrake@cshp.ca) et l'on vous mettra en contact avec la personne appropriée. Vous pouvez aussi indiquer aux réviseurs l'approche que vous souhaitez adopter (en la justifiant) et laisser le soin aux rédacteurs de trancher.

Bon nombre des articles resoumis par suite d'un avis de décision de catégorie $\mathrm{n}^{\circ} 3$ ne sont pas retournés aux réviseurs. Bien souvent, particulièrement si vous avez tenu compte de tous les points émis dans l'avis de décision original, votre manuscrit retravaillé ne sera révisé que par le rédacteur adjoint qui en a la responsabilité. Lorsque l'article revu est resoumis (le plus souvent) aux mêmes réviseurs ou (occasionnellement) à des réviseurs différents, rappelez-vous qu'ils mettront quelques fois le doigt sur des problèmes qui n’ont pas été détectés dans l'article originalement soumis. Bien que cela puisse vous exaspérer, souvenez-vous que nous travaillons dans votre intérêt et que nous voulons avoir les meilleurs articles qui soient dans notre Journal.

Même si vous recevez un avis de décision de catégorie $\mathrm{n}^{\circ} 4$ (rejeté après révision par les pairs), lisez attentivement les commentaires des réviseurs et mettez-les à profit pour mieux étoffer votre article avant de le présenter à un autre périodique. Parallèlement, vous ne devez pas oublier que le monde de la pharmacie est relativement petit et qu'un manuscrit que vous soumettez à un autre périodique peut finir dans les mains des mêmes réviseurs. En fait, cela s'est produit à au moins une occasion récemment; l'article en question avait été assigné à un rédacteur adjoint du JCPH qui l'avait révisé précédemment pour le compte d'un autre périodique. Mais à sa grande surprise, les auteurs avaient ignoré les commentaires pourtant minutieusement ficelés lors de la première révision, et l'article avait été soumis à l'identique au $J C P H$ qui l'a rejeté pour les mêmes raisons.

Nous gardons un registre des raisons motivant le rejet des manuscrits et voici les plus courantes : contient une erreur fatale de conception ou de présentation des données (p. ex., méthodologie ou analyses inappropriées), manque d'originalité ou ajoute peu d'information nouvelle à la littérature actuelle, a 
peu de portée ou de pertinence pour d'autres établissements, ne répond pas à la question faisant l'objet de la recherche, ne satisfait pas aux normes de pratique des pharmaciens canadiens, est mal rédigé (p. ex., manque d'organisation ou de clarté, description et interprétation inadéquates des résultats) et ne répond pas aux exigences du journal relativement à la soumission des articles.

Williams ${ }^{3}$ présente un excellent résumé sur la façon de répondre aux réviseurs : " de façon exhaustive, polie et factuelle ». En outre, pour faire écho aux propos de DeMaria ${ }^{4}$, le JCPH accorde de la valeur aux manuscrits " qui sont soumis sans délais, avec des réponses détaillées à chaque question soulevée et les changements au manuscrit apportés en réponse à chaque commentaire clairement soulignés dans la lettre de réplique. » Mais ce sont peut-être Peh et $\mathrm{Ng}^{5}$ qui résument le mieux le sujet : "Une demande de révision doit être reçue positivement, car cela signifie qu'il y a une possibilité que le manuscrit soit encore publiable, pourvu que tous les commentaires du rédacteur responsable et des réviseurs aient été pris en compte. »

\section{References}

1. Ensom MHH. Faits et comparaisons : Mises à jour concernant le Journal et indicateurs de performance [éditorial]. Can J Hosp Pharm 2010;63(4):285-287.

2. Ensom MHH. The art and science of peerless peer review [résumé]. Can J Hosp Pharm 2007;60(Suppl 1):31-32.
3. Williams HC. How to reply to referees' comments when submitting manuscripts for publication. J Am Acad Dermatol 2004;51(1):79-83.

4. DeMaria A. Manuscript revision. J Am Coll Cardiol 2011;57(25): 2540-2541.

5. Peh WC, $\mathrm{Ng} \mathrm{KH}$. Dealing with returned manuscripts. Singapore Med J 2009;50(11):1050-1052.

Mary H H Ensom, Pharm.D., FASHP, FCCP, FCSHP, FCAHS, est professeure et directrice, Programme de doctorat en pharmacie, Faculté des sciences pharmaceutiques, et boursière universitaire distinguée, University of British Columbia, et spécialiste en pharmacie clinique, Children's \& Women's Health Centre of British Columbia, Vancouver (Colombie-Britannique). Elle est également rédactrice en chef du JCPH.

\section{Adresse de correspondance :}

Dr Mary H H Ensom

Department of Pharmacy (OB7)

Children's \& Women's Health Centre of British Columbia

4500 Oak Street

Vancouver (Colombie-Britannique) V6B 6E4

Courriel : ensom@mail.ubc.ca

Remerciements

Je saisis cette occasion pour remercier très sincèrement toutes les personnes qui donnent bénévolement de leur temps en qualité de rédacteurs adjoints ou de réviseurs pour le $J C P H$. 